\title{
Structures of Alkaloid Biosynthetic Glucosidases Decode Substrate Specificity
}

\author{
Liqun Xia, ${ }^{1 *}$ Martin Ruppert, ${ }^{2 *}$ Meitian Wang, ${ }^{3 \#}$ Santosh Panjikar, ${ }^{4 \#}$ Haili Lin, ${ }^{1}$ Chitra \\ Rajendran, ${ }^{3}$ Leif Barleben, ${ }^{5}$ Joachim Stöckigt, ${ }^{1 \#}$ \\ ${ }^{1}$ Institute of Materia Medica, College of Pharmaceutical Sciences, Zhejiang University, 866 Yu Hang Tang \\ Road, Hangzhou 310058, P.R. China \\ ${ }^{2}$ Lehrstuhl für Pharmazeutische Biologie, Institut für Pharmazie und Biochemie, Johannes Gutenberg- \\ Universität, Staudinger Weg 5, D-55099 Mainz, Germany \\ ${ }^{3}$ Swiss Light Source, Paul Scherrer Institute, CH-5232 Villigen, Switzerland \\ ${ }^{4}$ Australian Synchrotron, 800 Blackburn Road, Clayton VIC, Australia 3168 \\ ${ }^{5}$ Department of Medical Biochemistry and Biophysics, Karolinska Institute, SE-17177 Stockholm, Sweden
}

"these authors contributed equally to this work

\#correspondence should be addressed to M. W. (meitian.wang@psi.ch); S. P. (panjikar.santosh@embl-

hamburg.com) and J. S. (joesto2000@yahoo.com)

Inventory of Supplemental Information:

Figure S1, The Role of RG as Tool for Facile Production of Small Alkaloid Libraries Including Novel Alkaloids with Un-natural C-2 Configuration by Chemo-enzymatic Approaches.

Figure S2A, Structure Based Alignment of $\beta$-Glucosidases of Family 1 from Higher Plants.

Figure S2B, Sequence-based Phylogenetic Tree of 11 Structurally Characterized Plant GH1 Enzymes.

Figure S3, Stereoview of Raucaffricine Binding Site of RG.

Figure S4, The Different Conformations of Glu476 in RG and Glu472 in SG.

Figure S5, Hydrogen Binding Network Between Secologanin and Residues in a Distance of $\leq 3.8 \AA$ in the Ligand Structure of $\mathrm{His}_{6}$-RG Inactive Mutant E186Q.

Table S1, Physical and Biochemical Properties of RG and SG from R. serpentina.

Table S2, Kinetic Constants of His $_{6}$-tagged RG Wild-type, Mutant W392A.

Table S3, Data Collection and Refinement Statistics of RG.

Supplemental Experimental Procedures 


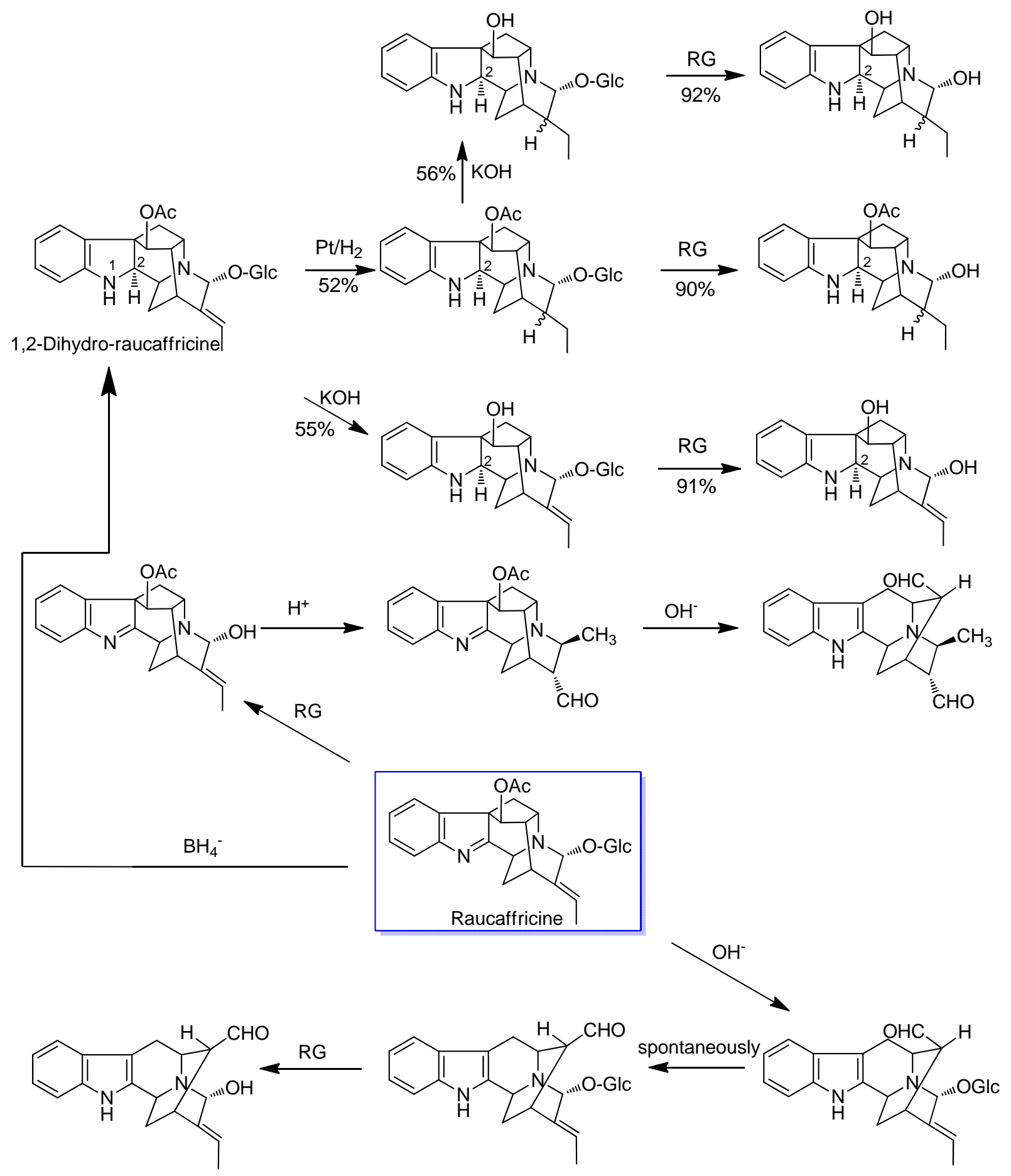

Figure S1, The Role of RG as Tool for Facile Production of Small Alkaloid Libraries Including Novel Alkaloids with Un-natural C-2 Configuration by Chemo-enzymatic Approaches. 
RG

RG

SG

CBG

Os3bglu7

Os 3bglu6

Zm-p60.1

Glu1

Dhr1

TaGlulb
ScGlu

$R G$

RG

CBG

Os 3bglu12

Os 3 bglu7

Zm-p60.1

Glu1

Dhr1

TaGlulb

RG

RG

RG

CBG

Os3bglu12

Os 3 bglu7

Os3bglu6

Zm-p60.1

Glu1

TaGlulb

RG

SG

Os 3bglu12

Os 3 bglu7

Os 3bglu6

Zm-p60.1

Glu1

Dhrl

TaGlulb
ScGlu

$\eta$

110

MATQSSAVIDSND .....AT MDNTOAEPLVVAIVPKPNASTEHTNSHLI PVT IHAFKPLP.
MAAAGAM PGLLLTELLLAVVIASGAYNG. MAARRANCALVLVLALALLAARDAGAAAVPK... MAPL ............................ MAPLLAAAMNHAAAH P G LRS HLVGPNNES F SRH HLP S S SPQS S KRRCNLSFTTR SARVGSQ.NG. VQMI MAL MALLUASAT MALLAATLNPTTHLS. LRSR. AGRNSENLWLRSTASS. KSKGRFCNLTIRAGTPSKPAEPIGPVFTKL
MALIGGTLPTTHLS. LRSR. AGRNSENVWLRSAASS. QTSKGRFCNLTVRAGTPSKPSEPIGPVFTKL

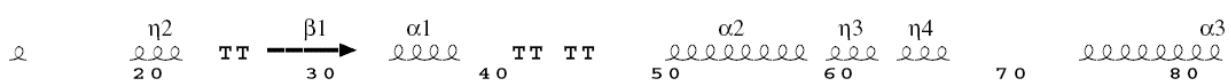

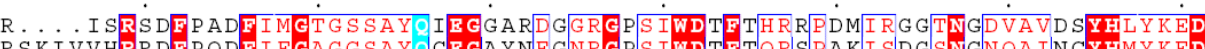

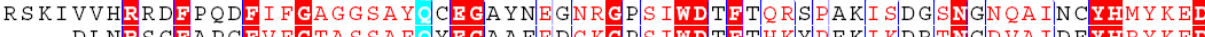

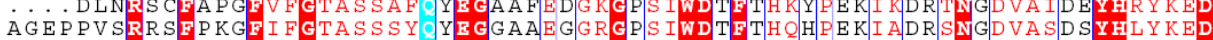

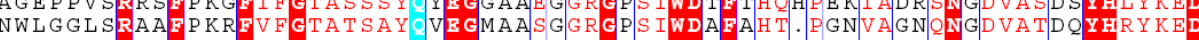
QSGGGLTRGSEPEGEVFGTASAAY Y Y EAVKED GRGQT IWDTEA H T. FGKITIDF SNADVAVDQYHRFEED

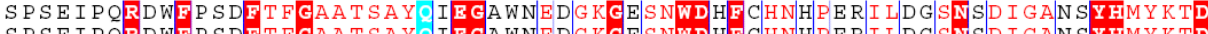

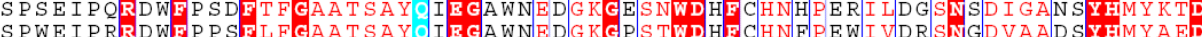

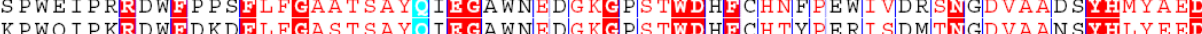

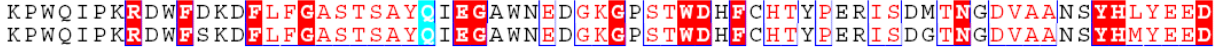

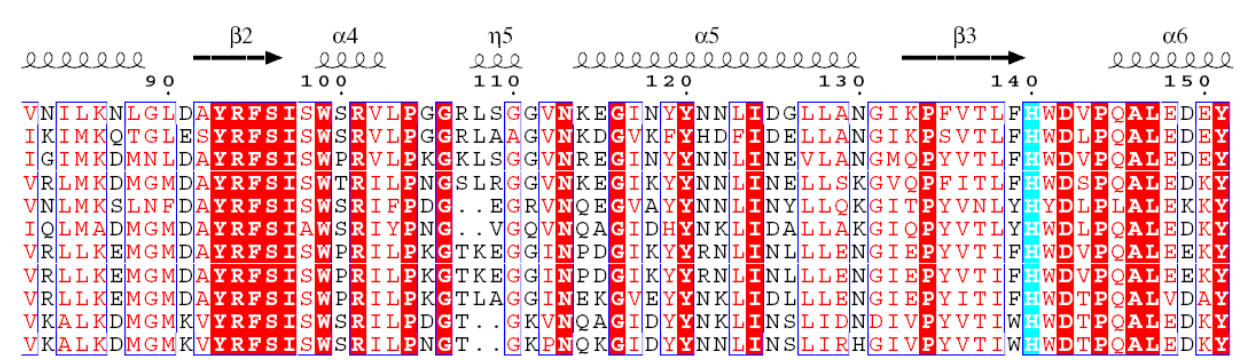

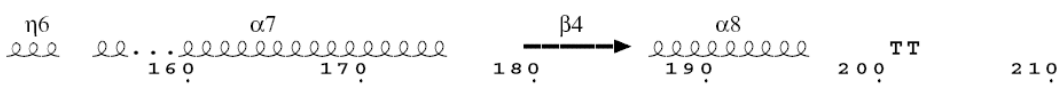

GGFLSPRI . . . VDDFCEYAELCEWEFGDRVEHWMTLNEPWTF SVHGYATGLY APGRGRTSPEHVNHPTVQ

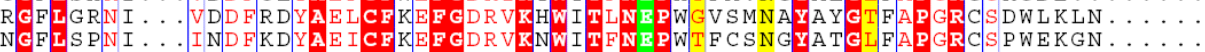

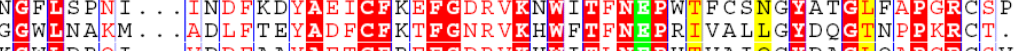

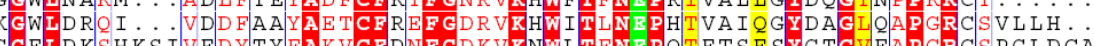

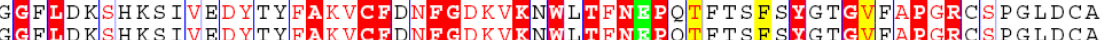

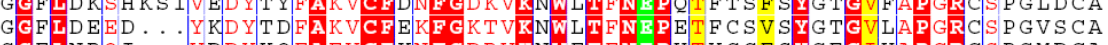

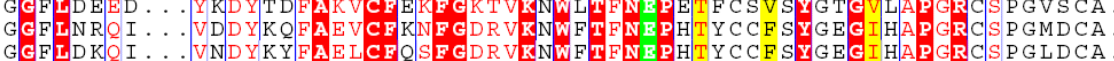

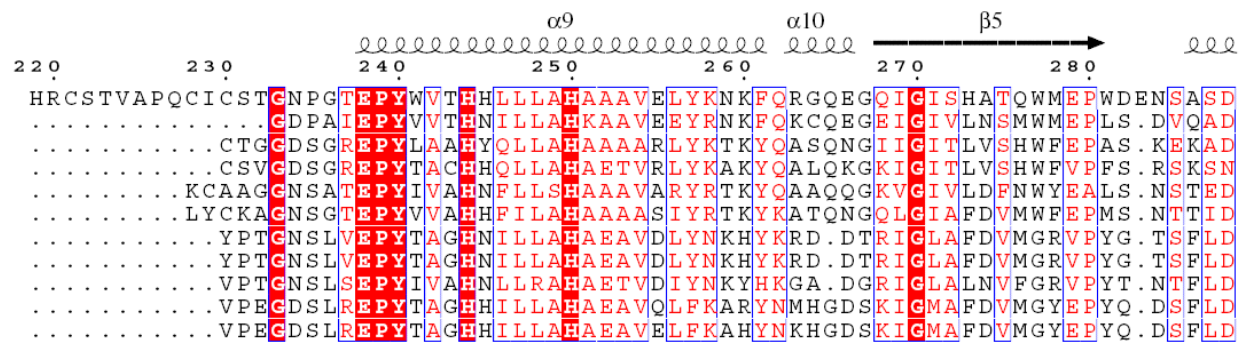

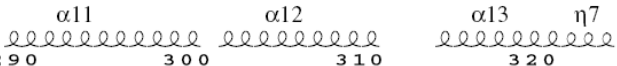

elelele

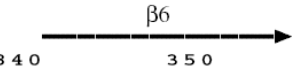

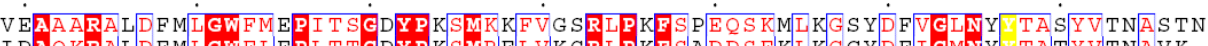

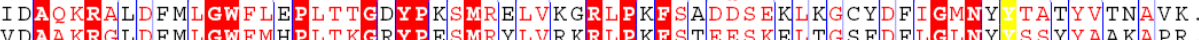

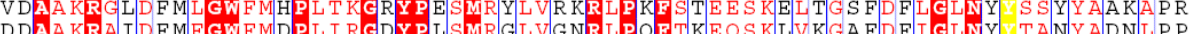

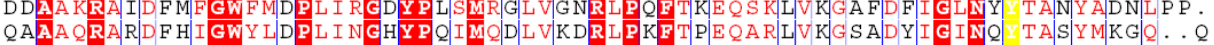

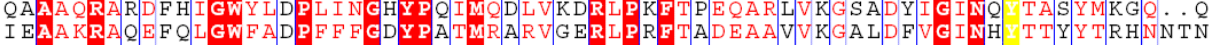

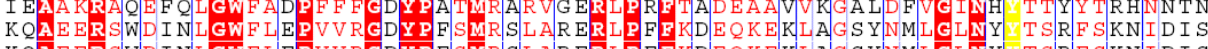

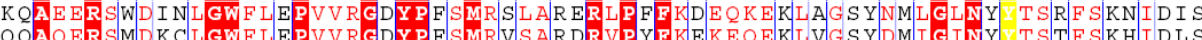

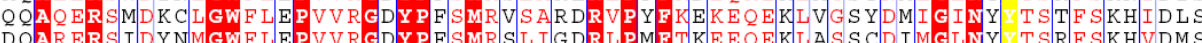

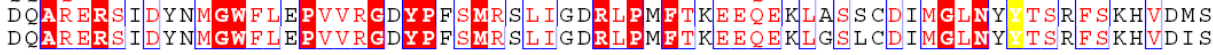





Figure S2A. Structure Based Alignment of $\beta$-Glucosidases of Family 1 from Higher Plants.

RG, raucaffricine glucosidase from Rauvolfia serpentina; SG, strictosidine glucosidase from Rauvolfia serpentina; CBG, cyanogenic $\beta$-glucosidase from Trifolium repens; Os3bglu12, Os3bglu7 and Os3bglu6 are $\beta$-glucosidases from Oryza sativa Japonica Group; Zm-p60.1 and, zeatin $\beta$-glucosidase from Zea mays; Glu1, $\beta$-glucosidase 1 from Zea mays; Dhr1, dhrurrinase 1 from Sorghum bicolor; TaGlulb, $\beta$-glucosidase from Triticum aestivum; ScGlu, $\beta$-glucosidase from Secale cereale. The green color indicates the conserved catalytic residues: Glu186, Glu420. The cyan color shows the five residues with hydrophilic interaction to the glucose part. The residues influencing the conformation of the aglycone part are marked in yellow. The residues colored in red are highly conserved residues of all $11 \beta$-glucosidases. 


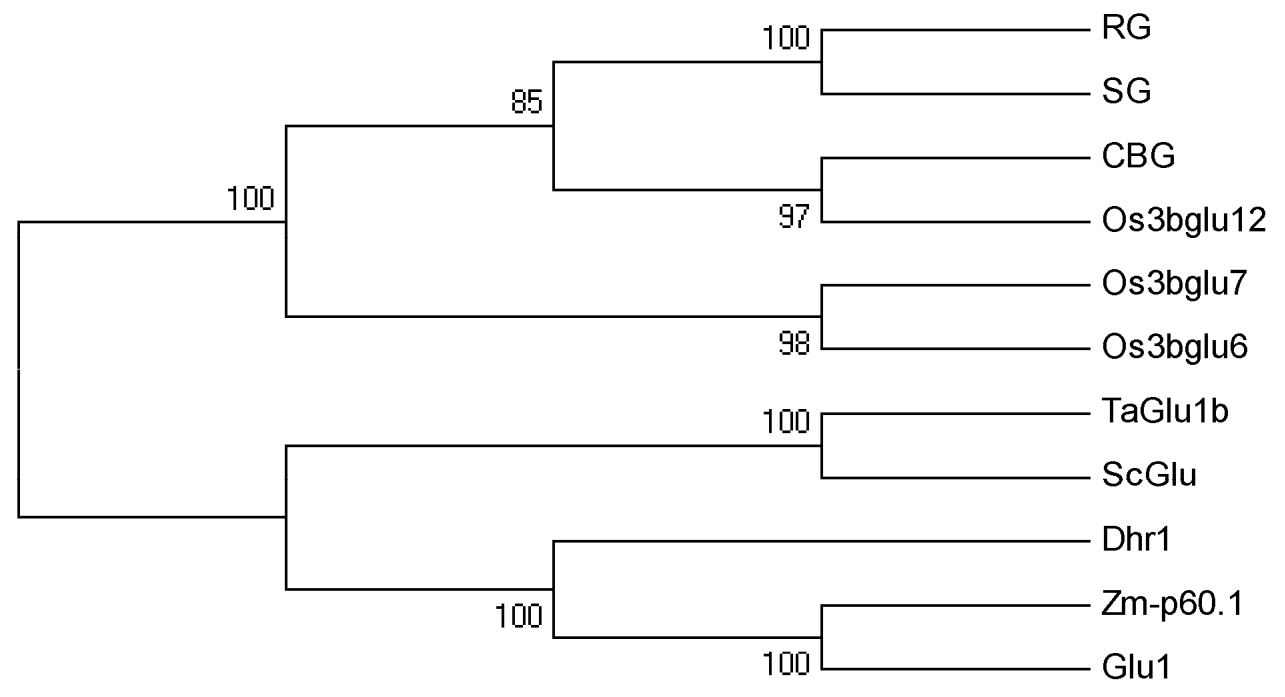

Figure S2B. Sequence-based Phylogenetic Tree of 11 Structurally Characterized Plant GH1 Enzymes.

The evolutionary history was inferred using the Neighbor-Joining method (Saitou and Nei, 1987). The optimal tree with the sum of branch length $=1.94776883$ is shown. The percentage of replicate trees in which the associated taxa clustered together in the bootstrap test (500 replicates) are shown next to the branches (Felsenstein, 1985). The evolutionary distances were computed using the p-distance method (Nei and Kumar, 2000) and are in the units of the number of amino acid differences per site. The analysis involved 11 amino acid sequences. All positions containing gaps and missing data were eliminated. There were a total of 458 positions in the final dataset. Evolutionary analyses were conducted in MEGA5 (Tamura et al., 2011). Enzymes were named according to their abbreviations used in Figure S2A.

Ab
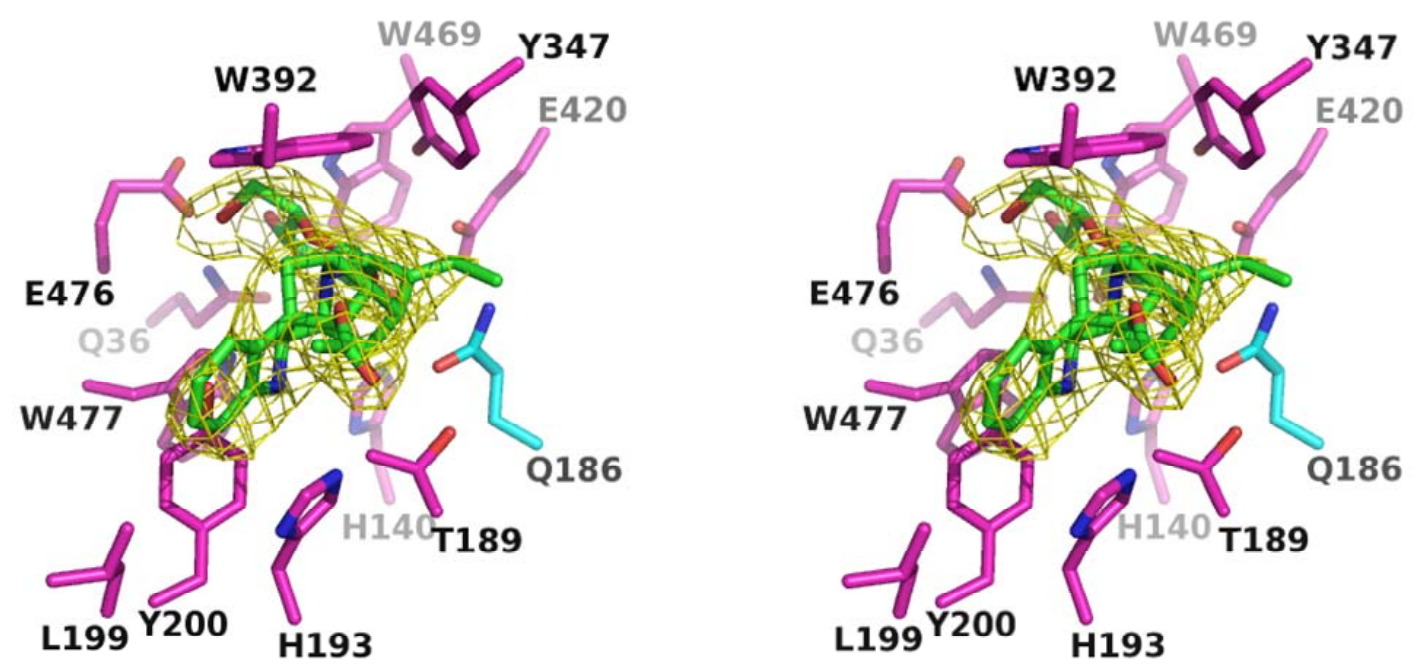

Figure S3. Stereoview of Raucaffricine Binding Site of RG. 


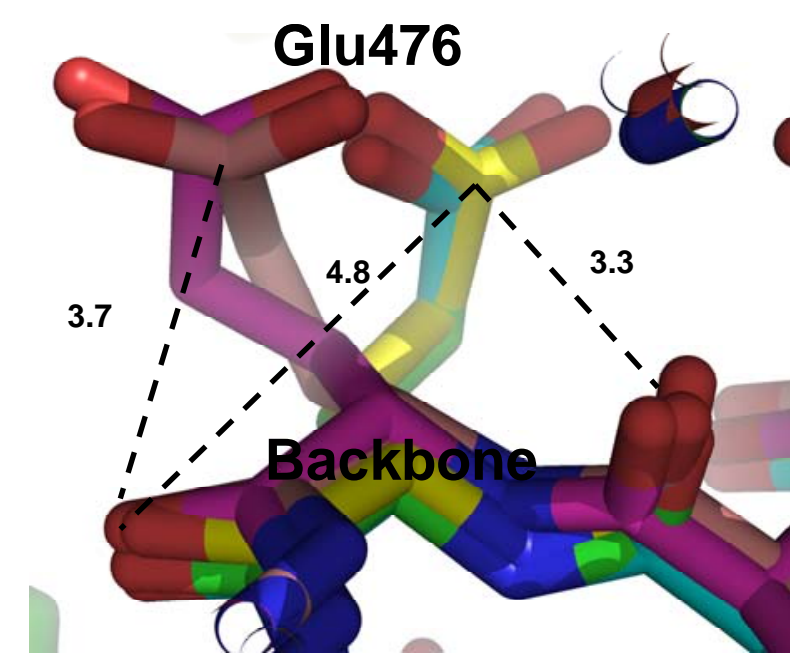

Figure S4. The Different Conformations of Glu476 in RG and Glu472 in SG.

The 3-D structure of RG-WT (magenta), RG-E186Q-dihydroraucaffricine complex (green), RG-E186Qsecologanin complex (cyan), SG-WT (yellow), SG-E207Q (salmon) was superimposed.

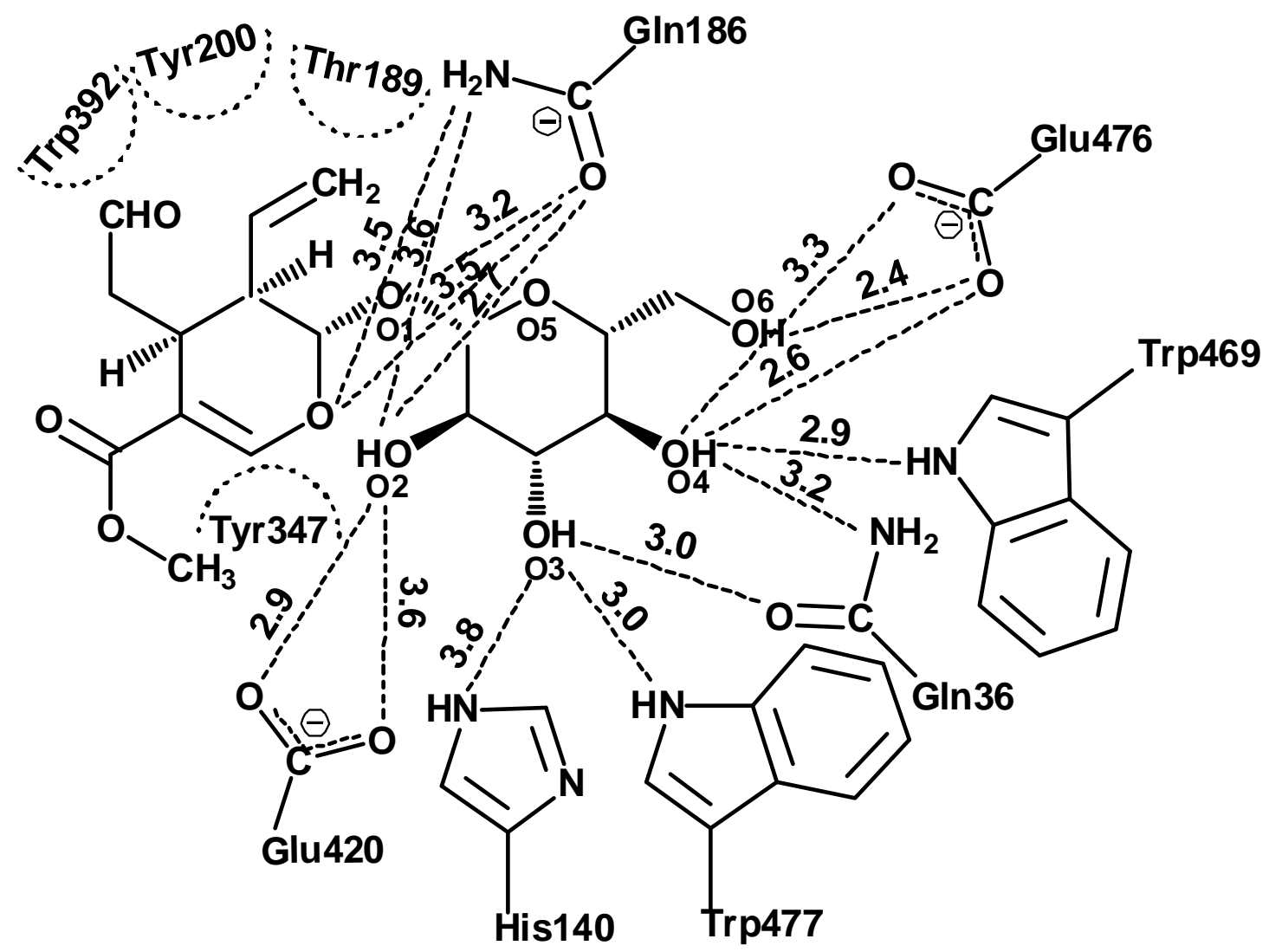

Figure S5. Hydrogen Binding Network Between Secologanin and Residues in a Distance of $\leq 3.8 \AA$ in the Ligand Structure of His $_{6}-$ RG Inactive Mutant E186Q. 
Table S1. Physical and Biochemical Properties of RG and SG from R. serpentina.

\begin{tabular}{|c|c|c|c|c|}
\hline & \multicolumn{2}{|c|}{$\mathbf{R G}$} & \multicolumn{2}{|c|}{ SG } \\
\hline Amino Acids & \multicolumn{2}{|c|}{540} & \multicolumn{2}{|c|}{532} \\
\hline Theoretical pI / rel. MW (Da) & \multicolumn{2}{|c|}{$6.07 / 60934.19$} & \multicolumn{2}{|c|}{$6.01 / 60880.59$} \\
\hline CAZY-Family & \multicolumn{2}{|c|}{ GH-1 } & \multicolumn{2}{|c|}{ GH-1 } \\
\hline Fold & \multicolumn{2}{|c|}{$(\beta / \alpha)_{8}$ barrel fold } & \multicolumn{2}{|c|}{$\begin{array}{l}(\beta / \alpha)_{8} \text { barrel fold (Barleben et al., } \\
2007)\end{array}$} \\
\hline Sequence-Identity / Similarity & \multicolumn{4}{|c|}{$55 \% / 69 \%$} \\
\hline \multirow{20}{*}{ Amino acid composition, } & Ala (A) 32 & $5.9 \%$ & Ala (A) 35 & $6.6 \%$ \\
\hline & $\operatorname{Arg}(\mathrm{R}) 29$ & $5.4 \%$ & $\operatorname{Arg}(\mathrm{R}) 25$ & $4.7 \%$ \\
\hline & $\operatorname{Asn}(\mathrm{N}) 33$ & $6.1 \%$ & Asn (N) 24 & $4.5 \%$ \\
\hline & Asp (D) 36 & $6.7 \%$ & Asp (D) 33 & $6.2 \%$ \\
\hline & Cys (C) 5 & $0.9 \%$ & Cys (C) 7 & $1.3 \%$ \\
\hline & Gln (Q) 14 & $2.6 \%$ & Gln (Q) 17 & $3.2 \%$ \\
\hline & Glu (E) 27 & $5.0 \%$ & Glu (E) 39 & $7.3 \%$ \\
\hline & Gly (G) 47 & $8.7 \%$ & Gly (G) 41 & $7.7 \%$ \\
\hline & His $(\mathrm{H}) 16$ & $3.0 \%$ & His $(\mathrm{H}) 16$ & $3.0 \%$ \\
\hline & Ile (I) $\quad 28$ & $5.2 \%$ & Ile (I) $\quad 28$ & $5.3 \%$ \\
\hline & Leu (L) 37 & $6.9 \%$ & Leu (L) & $6.4 \%$ \\
\hline & Lys (K) 26 & $4.8 \%$ & Lys $(\mathrm{K}) \quad 38$ & $7.1 \%$ \\
\hline & Met (M) 12 & $2.2 \%$ & $\operatorname{Met}(\mathrm{M}) \quad 9$ & $1.7 \%$ \\
\hline & Phe (F) 24 & $4.4 \%$ & Phe (F) 28 & $5.3 \%$ \\
\hline & Pro (P) 25 & $4.6 \%$ & Pro (P) 23 & $4.3 \%$ \\
\hline & Ser (S) 44 & $8.1 \%$ & Ser (S) 30 & $5.6 \%$ \\
\hline & Thr (T) 29 & $5.4 \%$ & Thr (T) 28 & $5.3 \%$ \\
\hline & $\operatorname{Trp}(\mathrm{W}) 14$ & $2.6 \%$ & $\operatorname{Trp}(\mathrm{W}) 12$ & $2.3 \%$ \\
\hline & Tyr (Y) 28 & $5.2 \%$ & Tyr (Y) 30 & $5.6 \%$ \\
\hline & Val (V) 34 & $6.3 \%$ & Val (V) 35 & $6.6 \%$ \\
\hline pH- / Temperature- Optimum & \multicolumn{2}{|c|}{$5.0 / 28^{\circ} \mathrm{C}$ (Warzecha et al., 2000) } & \multicolumn{2}{|c|}{$\begin{array}{l}5.0 / 30^{\circ} \mathrm{C} \text { (Gerasimenko et al., } \\
2002)\end{array}$} \\
\hline Km-value (strictosidine) & \multicolumn{2}{|c|}{$1.8 \mathrm{mM}$ (Warzecha et al., 2000) } & \multicolumn{2}{|c|}{$\begin{array}{l}0.12 \mathrm{mM} \text { (Gerasimenko et al., } \\
2002)\end{array}$} \\
\hline Km-value (raucaffricine) & \multicolumn{2}{|c|}{$1.3 \mathrm{mM}$ (Warzecha et al., 2000) } & \multicolumn{2}{|c|}{ No product detectable } \\
\hline
\end{tabular}


Table S2. Kinetic Constants of His $_{6}$-tagged RG Wild-type, Mutant Trp392Ala.

\begin{tabular}{lllrl}
\hline Enzyme & Substrate & $\mathrm{K}_{\mathrm{m}}(\mathrm{mM})$ & \multicolumn{1}{c}{$\mathrm{K}_{\mathrm{cat}}\left(\mathrm{s}^{-1}\right)$} & $\mathrm{K}_{\mathrm{cat}} / \mathrm{K}_{\mathrm{m}}\left(1 /\left(\mathrm{mM} \mathrm{s}^{-1}\right)^{*}\right.$ \\
\hline \multirow{2}{*}{ RG-WT } & Raucaffricine* & $0.78 \pm 0.08$ & $47.11 \pm 8.70$ & 59.94 \\
& Strictosidine* & $1.70 \pm 0.03$ & $1.26 \pm 0.28$ & 0.72 \\
\hline \multirow{2}{*}{ RG-Trp392Ala } & Raucaffricine & $3.10 \pm 1.49$ & $1.11 \pm 0.86$ & 0.35 \\
& Strictosdine & $3.10 \pm 0.6$ & $3.74 \pm 1.31$ & 1.17 \\
\hline
\end{tabular}

Measurements of RG-WT and RG-Trp392Ala mutant for conversion of the substrates raucaffricine and strictosidine (average data from $2 *$ or 3 different measurements) are displayed.

Table S3. Data Collection and Refinement Statistics of RG.

\begin{tabular}{|c|c|c|c|c|}
\hline \multirow{2}{*}{$\begin{array}{l}\text { Measurement temperature } \\
\text { Data set }\end{array}$} & \multirow{2}{*}{$\begin{array}{l}\text { Cryo-temperature } \\
(100 \mathrm{~K}) \\
\text { RG-WT-glycerol } \\
\text { Complex }\end{array}$} & \multicolumn{3}{|c|}{ Room temperature $(295 \mathrm{~K})$} \\
\hline & & RG-E186Q & $\begin{array}{l}\text { RG-E186Q-1,2- } \\
\text { dihydro- } \\
\text { raucaffricine } \\
\text { Complex }\end{array}$ & $\begin{array}{c}\text { RG-E186Q- } \\
\text { secologanin } \\
\text { Complex }\end{array}$ \\
\hline Wavelength $(\AA)$ & 0.8150 & 1.0000 & 1.0000 & 1.0000 \\
\hline Unit cell $(\AA)$ & $a=102.77$ & $a=105.56$ & $a=104.89$ & $a=104.93$ \\
\hline (Space group I222) & $b=127.33$ & $b=129.52$ & $b=129.55$ & $b=129.20$ \\
\hline & $C=215.84$ & $C=216.64$ & $c=216.92$ & $C=216.80$ \\
\hline Total reflections & 1169406 & 254774 & 279283 & 332690 \\
\hline Unique reflections & 76299 & 75161 & 55224 & 65973 \\
\hline Redundancy & 15.3 & 3.4 & 5.1 & 5.1 \\
\hline Mosaicity & 0.43 & 0.13 & 0.18 & 0.30 \\
\hline Resolution $(\AA)$ & $20-2.15$ & $\begin{array}{l}94.89-2.20 \\
(2.32-2.20)\end{array}$ & $\begin{array}{l}65.16-2.43(2.56- \\
2.43)\end{array}$ & $\begin{array}{l}47.68-2.30(2.42- \\
2.30)\end{array}$ \\
\hline Completeness (\%) & $99.3(98.8)^{\mathrm{a}}$ & $99.5(98.8)$ & $98.4(91.0)$ & $99.6(97.6)$ \\
\hline $\mathrm{I} / \sigma(\mathrm{I})$ & $24.6(4.4)$ & $10.0(2.7)$ & $11.7(2.9)$ & $16.1(3.4)$ \\
\hline$R_{\text {merge }}(\%)^{\mathrm{b}}$ & $11.7(69.4)$ & $7.0(39.6)$ & $8.5(48.5)$ & $6.5(49.0)$ \\
\hline Refinement & & & & \\
\hline Resolution $(\AA)$ & $20-2.15$ & $94.89-2.20$ & $65.16-2.43$ & $47.68-2.30$ \\
\hline$R_{\text {cryst }} / R_{\text {free }}(\%)^{\mathrm{c}}$ & $16.5 / 20.4$ & $22.0 / 26.7$ & $20.6(23.8)$ & $19.1(23.1)$ \\
\hline Av. B $\left(\AA^{2}\right)$ for protein & 36.8 & 39.0 & 34.6 & 40.9 \\
\hline Av. B $\left(\AA^{2}\right)$ for water & 26.1 & 41.2 & 32.4 & 42.2 \\
\hline $\begin{array}{l}\text { Av. B }\left(\AA^{2}\right) \text { for substrate }{ }^{1} \\
\text { Number of atoms }\end{array}$ & 35.7 & - & 59.2 & 76.0 \\
\hline Non-hydrogen & 8016 & 7686 & 7719 & 7737 \\
\hline
\end{tabular}




\begin{tabular}{|c|c|c|c|c|}
\hline Water & 443 & 153 & 93 & 153 \\
\hline \multicolumn{5}{|l|}{ R.m.s.deviations } \\
\hline Bond $(\AA)$ & 0.023 & 0.016 & 0.013 & 0.012 \\
\hline Angles $\left({ }^{\circ}\right)$ & 1.69 & 1.69 & 1.43 & 1.34 \\
\hline \multicolumn{5}{|l|}{ Ramachandran plot } \\
\hline $\begin{array}{l}\text { Residues in most favorable } \\
\text { regions (\%) }\end{array}$ & 90.3 & 92.0 & 93.5 & 94.2 \\
\hline $\begin{array}{l}\text { Residues in additional } \\
\text { allowed regions (\%) }\end{array}$ & 8.7 & 6.5 & 5.5 & 4.4 \\
\hline $\begin{array}{l}\text { Residues in generously } \\
\text { allowed regions (\%) }\end{array}$ & 1.0 & 1.5 & 1.0 & 1.4 \\
\hline PDB code & $4 \mathrm{~A} 3 \mathrm{Y}$ & $3 \mathrm{U} 5 \mathrm{U}$ & $3 \mathrm{U} 57$ & $3 \mathrm{U} 5 \mathrm{Y}$ \\
\hline
\end{tabular}

\section{Supplemental Experimental Procedures}

\section{Expression, Purification, Activity Assay, Kinetic Data and Mutagenesis of RG}

In order to obtain recombinant RG [AAF03675], the cDNA of RG (AF149311), isolated by Warzecha et al. (2000) from R. serpentina, was cloned into the pQE-2 vector as a fusion protein with an N-terminal His ${ }_{6}$-tag (Ruppert et al., 2006). The pQE-2 plasmid construct was transformed into E. coli strain M15 [pREP4] (Qiagen, Hilden, Germany), expressed, isolated and purified as described by Ruppert et al. (2006). RG concentration was determined by the Bradford method (1976) except the protein concentration for RG enzyme activity assays was determined at $\mathrm{A}_{280 \mathrm{~nm}}$ with Warburg equation and the purity of RG was analyzed by SDS-PAGE.

For determination of relative enzyme activity, the consumption of raucaffricine, strictosidine, secologanin and arbutin were monitored at $258 \mathrm{~nm}, 280 \mathrm{~nm}, 236 \mathrm{~nm}, 284 \mathrm{~nm}$ respectively. Quantitative RG kinetic assays were performed at the optimal $\mathrm{pH}$ of the enzyme ( $\mathrm{pH} \mathrm{5,} \mathrm{sodium} \mathrm{citrate).} \mathrm{The} \mathrm{standard} \mathrm{curve} \mathrm{of} \mathrm{raucaffricine} \mathrm{and}$ strictosidine was determined at concentrations of $0.4,0.8,1.2,1.6,2.0 \mathrm{mM}$, and the $R^{2}$ values were up to 0.99 . In a total volume of $100 \mu \mathrm{l}, 0.11 \mu \mathrm{g}$ wild-type $\mathrm{RG}$ was incubated with raucaffricine $(1.6 \mathrm{mM})$ at 30 for $30 \mathrm{~min}$. Under these conditions enzyme activity was set at $100 \%$; an average from three measurements were used for each ligand. For RG mutants with a low activity and incubation with other putative substrates, enzyme amounts were increased to $35 \mu \mathrm{g} R \mathrm{R}$, leading to a detection limit of $<0.02 \%$ relative activity. $\mathrm{K}_{\mathrm{m}}$ values were determined in a similar way, with enzyme concentrations depending on the activity of the mutant from 0.035 to $7 \mu \mathrm{g}$ and substrate concentrations between 0.015 and $2 \mathrm{mM}$. Kinetic data, $\mathrm{K}_{\mathrm{m}}$ and $\mathrm{V}_{\max }$ were determined by HPLC with a 
LiChrospher 250 x $4 \mathrm{~mm}, 5 \mu \mathrm{m} 60 \mathrm{RP}$-select B column, injection volume of $0.05 \mathrm{ml}$ and flow rate of $1.0 \mathrm{ml}$ x $\min ^{-1}$.

Site-directed mutants were constructed using the QuickChange ${ }^{\mathrm{TM}}$ Site-Directed Mutagenesis Kit (Stratagene, La Jolla, USA) and RG-pQE-2 plasmid as a template. Mutants were verified by sequencing, expressed and purified in the same way as the wild type enzyme. The primer pairs used for mutations are shown in the following table.

Primer Pairs used for Generating 12 Different Mutants.

\begin{tabular}{|c|c|}
\hline Mutant & Primer pairs \\
\hline \multirow[t]{2}{*}{ E186D } & Glu186Asp-for: 5'-GATGACATTGAACGACCCATGGACATTTAGC-3' \\
\hline & 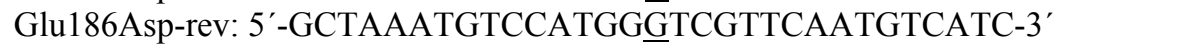 \\
\hline \multirow[t]{2}{*}{ E186Q } & Glu186Gln-for: 5'-GATGACATTGAACCAA $\overline{G C C A T G G A C A T T T A G C-3 ' ~}$ \\
\hline & Glu186Gln-rev: 5'-GCTAAATGTCCATḠGCTGGTTCAATGTCATC-3' \\
\hline \multirow[t]{2}{*}{ E420Q } & Glu420Gln-for: 5'-CATATATGTCACACAGAĀTGGAGTTGACG-3' \\
\hline & Glu420Gln-rev: 5'-CGTCAACTCCATT $\overline{\mathrm{CTG} T G T G A C A T A T A T G-3 ' ~}$ \\
\hline \multirow[t]{4}{*}{ E186Q/E420Q } & 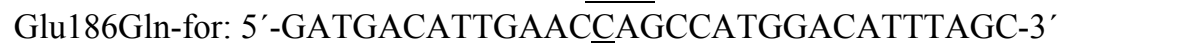 \\
\hline & Glu186Gln-rev: 5'-GCTAAATGTCCATḠGCTGGTTCAATGTCATC-3' \\
\hline & Glu420Gln-for: 5'-CATATATGTCACACAGAĀTGGAGTTGACG-3' \\
\hline & 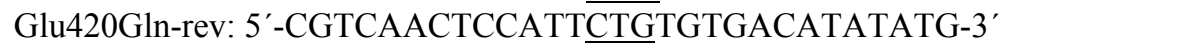 \\
\hline \multirow[t]{2}{*}{ W392A } & Trp392Ala-for: 5'-CCAGTCTGGTTCA $\overline{G A T G C G C T A C T T A T T T A T C C A G-3 ' ~}$ \\
\hline & Trp392Ala-rev: 5'-CTGGATAAATAAGTAGCGCATCTGAACCAGACTGG-3' \\
\hline \multirow[t]{2}{*}{ H193A } & His193Ala-for: 5’-CATGGACATTTAGCGTTGCTGGATATGCAACAGGC-3' \\
\hline & His193Ala-rev: 5'-GCCTGTTGCATATCCAGCAACGCTAAATGTCCATG-3' \\
\hline \multirow[t]{2}{*}{ Y200A } & Tyr200Ala-for: 5'-GATATGCAACAGGCCTTGCAGCACCCGGTCGAGGTC-3' \\
\hline & Tyr200Ala-rev: 5'-GACCTCGACCGGGTGCTGCAAGGCCTGTTGCATATC-3' \\
\hline \multirow[t]{2}{*}{ T189A } & Thr189Ala-for: 5'-GAACGAGCCATGGGCATTTAGCGTTCATGG-3' \\
\hline & Thr189Ala-rev: 5'-CCATGAACGCTAA $\overline{A T G C C C A T G G C T C G T T C-3 ' ~}$ \\
\hline \multirow[t]{2}{*}{$\mathrm{F} 485 \mathrm{Y}$} & Phe485Tyr-for: 5'-GGTATGGAGTGCGTTATGGTATCATCCACATCG-3' \\
\hline & Phe485Tyr-rev: 5'-CGATGTGGATGATACCATAACGCACTCCATACC-3' \\
\hline \multirow[t]{2}{*}{ S390G } & Ser390Gly-for:5'-GGTCCCCAGTCTGGTGGAGATTGGCTACTTATTTATC-3' \\
\hline & Ser390Gly-rev:5'-GATAAATAAGTAGCCAATCTCCACCAGACTGGGGACC-3' \\
\hline \multirow[t]{2}{*}{ E476L } & Glu476Leu-for:5'-CATTATTGGATAATTTTCTATGGGGTGAAGGGTATGG-3' \\
\hline & Glu476Leu-rev:5'-CCATACCCTTCACCCCATAGAAAATTATCCAATAATG-3' \\
\hline \multirow[t]{2}{*}{ E476A } & Glu476Ala-for:5'-CATTATTGGATAATTTTGCATGGGGTGAAGGGTATGG-3' \\
\hline & Glu476Ala-rev: 5'-CCATACCCTTCACCCCATGCAAAATTATCCAATAATG-3' \\
\hline
\end{tabular}

\section{Structural Analysis}

Structure-based sequence alignment was performed using the program STAMP (Russell and Barton, 1992) and the picture of the sequence alignment was made using the program ESPRIPT (Gouet et al, 1999). All figures were produced using MOLSCRIPT (Kraulis, 1991), RASTER3D (Merrit and Murphy, 1994), PyMol (DeLano, 2002) and LIGPLOT (Wallace et al., 1995). The 2D diagram was prepared using the program CHEMDRAW and edited using Microsoft Power point.

\section{Supplemental References}


Bradford, M.M. (1976). A rapid and sensitive method for the quantitation of microgram quantities of protein utilizing the principle of protein-dye binding. Anal. Biochem. 72, 248-254.

DeLano, W.L. (2002). The PyMOL Molecular Graphics System (San Carlos, CA: DeLano Scientific).

Felsenstein J. (1985). Confidence limits on phylogenies: An approach using the bootstrap. Evolution 39, 783-791.

Gerasimenko, I., Sheludko, Y., Ma, X. and Stöckigt, J. (2002). Heterologous expression of a Rauvolfia cDNA encoding strictosidine glucosidase, a biosynthetic key to over 2000 monoterpenoid indole alkaloids. Eur. J. Biochem. 269, 2204-2213.

Gouet, P., Courcelle, E., Stuart, D.I. and Metoz, F. (1999). ESPript: multiple sequence alignments in PostScript. Bioinformatics 15, 305-308.

Kraulis, P.J. (1991). Molscript: A program to produce both detailed and schematic plots of protein structures. J. Appl. Crystallogr. 24, 946-950.

Merrit, E.A. and Murphy, M.E. (1994). Raster3D Version 2.0. A program for photorealistic molecular graphics. Acta Crystallogr. D Biol. Crystallogr. 50, 869-873

Nei M. and Kumar S. (2000). Molecular Evolution and Phylogenetics. Oxford University Press, New York.

Ruppert, M., Panjikar, S., Barleben, L. and Stöckigt, J. (2006). Heterologous expression, purification, crystallization and preliminary X-ray analysis of raucaffricine glucosidase, a plant enzyme specifically involved in Rauvolfia alkaloid biosynthesis. Acta Crystallogr. Sect. F 62, 257-260.

Russell, R.B. and Barton, G.J. (1992). Multiple protein sequence alignment from tertiary structure comparison: Assignment of global and residue confidence levels. Protein Struct. Funct. Genet. 14, 309-323.

Saitou N. and Nei M. (1987). The neighbor-joining method: A new method for reconstructing phylogenetic trees. Molecular Biology and Evolution 4, 406-425.

Tamura K., Peterson D., Peterson N., Stecher G., Nei M., and Kumar S. (2011). MEGA5: Molecular Evolutionary Genetics Analysis using Maximum Likelihood, Evolutionary Distance, and Maximum Parsimony Methods. Molecular Biology and Evolution (In Press).

Wallace, A.C., Laskowski, R.A. and Thornton, J.M. (1995). LIGPLOT: A program to generate schematic diagrams of protein-ligand interactions. Protein Eng. 8, 127-134.

Warzecha, H., Gerasimenko, I., Kutchan, T.M. and Stöckigt, J. (2000). Molecular cloning and functional bacterial expression of a plant glucosidase specifically involved in alkaloid biosynthesis. Phytochemistry 54, 657-666. 